\title{
Brachial plexus dose tolerance in head and neck cancer patients treated with sequential intensity modulated radiation therapy
}

\author{
Tarita O Thomas ${ }^{1 \dagger}$, Tamer Refaat ${ }^{1,4,5 \dagger}$, Mehee Choi $^{1}$, lan Bacchus ${ }^{1}$, Sean Sachdev ${ }^{1}$, Alfred W Rademaker ${ }^{2}$, \\ Vythialingam Sathiaseelan ${ }^{1}$, Achilles Karagianis ${ }^{3}$ and Bharat B Mittal ${ }^{{ }^{*}}$
}

\begin{abstract}
Purpose: We aimed to study the radiation induced brachial plexopathy in patients with head and neck squamous cell carcinoma (HNSCC) treated with Sequential Intensity Modulated Radiation Therapy (S-IMRT).

Methods and materials: This IRB approved study included 68 patients with HNSCC treated consecutively. Detailed dose volume histogram data was generated for ipsilateral and contralateral brachial plexus (BP) volumes receiving a specified dose (Vds) i.e. V50-V75 and dose in Gray covering specified percent of BP volume (Dvs) i.e. D5-D30 and maximum point doses (Dmax). To assess BP injury all patients' charts were reviewed in detail for sign and symptoms of BP damage. Post-hoc comparisons were done using Tukey-Kramer method to account for multiple significance testing.

Results: The mean and maximum doses to BP were significantly different $(p<.05)$ based on tumor site, nodal status and tumor stage. The mean volume to the ipsilateral BP for V50, V60, V70, and V75 were $7.01 \mathrm{cc}, 4.37 \mathrm{cc}, 1.47 \mathrm{cc}$ and $0.24 \mathrm{cc}$, respectively. The mean dose delivered to $\leq 5 \%$ of ipsilateral BP was $68.70 \mathrm{~Gy}$ (median 69.5Gy). None of the patients had acute or late brachial plexopathy or any other significant neurological complications, with a minimum follow up of two years (mean 54 months).

Conclusions: In this study cohort, at a minimum of two-years follow up, the mean dose of 68.7Gy, a median dose to 69.5Gy to $\leq 5 \%$ of ipsilateral BP, and a median Dmax of 72.96Gy did not result in BP injury when patients were treated with S-IMRT technique. However, longer follow up is needed.
\end{abstract}

Keywords: Brachial plexus, Radiation, Tolerance

\section{Summary}

Radiation therapy may induce brachial plexopathy. Current Radiation Therapy Oncology Group (RTOG) guidelines suggest dose constraints ranging from 60-66Gy. We found that over a minimum follow-up of two years (mean 54 months) none of our patients developed acute or late brachial plexopathy with a mean, and median dose of $68.7 \mathrm{~Gy}$, and $69.5 \mathrm{~Gy}$ delivered to less than $5 \%$ of ipsilateral BP and a median maximum point dose of 72.96 Gy to the ipsilateral BP although longer follow-up is necessary.

\footnotetext{
* Correspondence: bmittal@nmh.org

${ }^{\dagger}$ Equal contributors

'Department of Radiation Oncology, Northwestern University, Robert H. Lurie Comprehensive Cancer Center, 251 East Huron, LC-178, Chicago, IL 60611, USA

Full list of author information is available at the end of the article
}

\section{Introduction}

Concomitant chemo-radiation (CRT) is the current standard of care for local-regional advanced head and neck squamous cell carcinoma (HNSCC). Intensity Modulated Radiation Therapy (IMRT) is the commonly utilized radiation therapy technique in this setting, either using Sequential IMRT (S-IMRT) [1] or Simultaneous Integrated Boost (SIB-IMRT) techniques [2].

Radiation induced brachial plexopathy is a concerning adverse event among HNSCC patients; it is defined as neurologic impairment of a transient or permanent nature as a sequela to radiation therapy [3]. Symptoms include paresthesia, pain, weakness, and motor dysfunction affecting the chest, shoulder and upper extremity [4]. The Radiation Therapy Oncology Group (RTOG) has endorsed an atlas that has been developed and validated for delineating

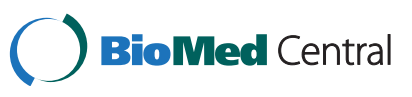

(c) 2015 Thomas et al.; licensee BioMed Central. This is an Open Access article distributed under the terms of the Creative Commons Attribution License (http://creativecommons.org/licenses/by/4.0), which permits unrestricted use, distribution, and reproduction in any medium, provided the original work is properly credited. The Creative Commons Public Domain Dedication waiver (http://creativecommons.org/publicdomain/zero/1.0/) applies to the data made available in this article, unless otherwise stated. 
the brachial plexus (BP), which has helped in standardizing $\mathrm{BP}$ contouring [5-7]. Limits on the dose to the $\mathrm{BP}$ in patients receiving IMRT have been recommended by the RTOG to 60-66 Gy on most RTOG clinical protocols including; RTOG 0435 ( $\leq 60$ Gy), RTOG 0522 ( $\leq 60$ Gy), RTOG 0412 (Dmax 60 Gy), and RTOG 0615 ( $\leq 66$ Gy). However, based on tumor location and stage, some patients may be treated to higher doses of radiation to the BP. Furthermore, radiation therapy induced brachial plexopathy may vary depending on dose per fraction, total dose and volume of BP exposed to radiation and the use of CRT [3]. Most of the published data regarding dose to the BP are based on 2D/3D or SIB-IMRT planning techniques. This is the first report evaluating the dose/volume relationship to radiation-induced brachial plexopathy in patients with locally advanced HNSCC treated with S-IMRT.

\section{Methods}

\section{Patient selection \& adverse event reporting}

The institutional review board of Northwestern University approved this study. From January 2003 to December 2008 a total of 68 patients with HNSCC were treated with S-IMRT. Patients were treated to a prescribed dose of 54-72 Gy with or without concurrent chemotherapy. Lower doses were prescribed to patients treated in adjuvant fashion. All patients had a minimum of two years follow-up. Brachial plexopathy was retrospectively determined according to the Common Terminology Criteria for Adverse Events version 3.0.

\section{Immobilization and simulation}

Patients were immobilized using an Aquaplast face mask (WFR/Aquaplast Corp, Wyckoff, New Jersey). Treatment planning CT scan was performed using IV contrast in the majority of patients. CT imaging included the region between the vertex and carina with $3 \mathrm{~mm}$ slice thickness.

\section{Target volumes definition}

The details of S-IMRT target volume definition and treatment planning have been reported [1]. The following guidelines were used:

\section{Definitive S-IMRT:}

The gross tumor volume (GTV) included clinically and/ or radiologically visible disease. Clinical target volume 1 $\left(\mathrm{CTV}_{1}\right)$ included GTV and high and low risks elective nodal regions in the neck with a $1-2 \mathrm{~cm}$ margin. Clinical target volume $2\left(\mathrm{CTV}_{2}\right)$ included GTV and high-risk elective nodal regions with $0.75-1.5 \mathrm{~cm}$ margin. Clinical target volume $3\left(\mathrm{CTV}_{3}\right)$ included GTV expanded by 0.5 $1 \mathrm{~cm}$ to cover any microscopic soft tissue extension.
Three planning target volumes (PTV 1 - 3) were created that encompassed the corresponding CTV with a margin of $3-5 \mathrm{~mm}$ to account for set up and patient movement errors. Later on, for sake of simplicity, we only outlined GTV and three PTVs that encompassed corresponding CTVs as defined above. We used axial images from the planning CT to identify nodal levels in the neck as described by Som et al. [8]. We largely used the historical data for neck metastases summarized by Chao et al. [9] to stratify nodal disease as high-risk and low-risk for elective neck irradiation.

\section{Post-operative S-IMRT:}

The surgical bed was defined as the area of preoperative GTV and the operated area that included the resected tumor, involved lymph nodes and post-surgical changes. $\mathrm{CTV}_{1}$ included the surgical bed and regional high and low risk elective nodal chain with 1-2 cm margin. $\mathrm{CTV}_{2}$ included surgical bed and high-risk elective nodal chain with $1-2 \mathrm{~cm}$ margin. In patients with highrisk features like extra-capsular extension (ECE) the surrounding soft tissue was included with a generous margin. Similar to definitive S-IMRT, each CTV was expanded by 3 - $5 \mathrm{~mm}$ to create $\mathrm{PTV}_{1}$ and $\mathrm{PTV}_{2}$ and later on for simplicity, the surgical bed and 2-3 PTVs were created. In both definitive as well as post-operative cases, the S-IMRT target volumes were drawn $2-3 \mathrm{~mm}$ deeper to the skin to spare dermal structures unless the gross tumor or ECE was very close to the skin.

\section{Dose specifications}

Radiation doses were sequentially prescribed using conventional fractionation of $180-200$ cGy to all patients. This study included only patients who received once a day, five-days a week, conventional fractionation and excluded patients who were treated using hyperfractionated or accelerated fractionation schemes. Typically, the low risk target volume $\left(\mathrm{PTV}_{1}\right)$ was treated to $45-50 \mathrm{~Gy}$, the intermediate to high-risk target volume $\left(\mathrm{PTV}_{2}\right)$ was treated to $56-66$ Gy and the gross tumor with expansion $\left(\mathrm{PTV}_{3}\right)$ was treated to $66-72$ Gy. Three separate IMRT plans corresponding to each PTV were generated and whole neck IMRT was used.

\section{Dose optimization}

Treatment planning utilized 7 - 9 beams inverse planning IMRT technique. For all target PTVs, maximum dose was used to restrict dose to $<110 \%$ of the prescribed dose, whereas maximum dose-volume histograms (DVH) was used to restrict the $105 \%$ dose volume inside PTV and minimum DVH used to ascertain that at least $95 \%$ of the PTV is covered by $100 \%$ of the prescribed dose. 
For normal structures, a combination of max dose, max DVH, and max Equivalent Uniform Dose were used as optimization criteria. To achieve a clinically acceptable plan, the objective values and weights were iteratively adjusted with the following order of priority: (1) minimize the maximum dose to the serial organs such as the spinal cord and brainstem; (2) minimize the mean dose to the swallowing organs at risk (OARs) such as the pharyngeal constrictors, larynx, postcricoid esophagus, as well as BP; and (3) minimize the mean dose to parotids and oral cavity.

\section{Computational method for analysis of dose to BP}

For each patient, the Pinnacle Treatment Planning system was used to generate a text file that contained DVH data, PTV volumes, and treatment plan statistics. MATLAB (version 2012a) was then used to format and parse the data into an Excel workbook for further analysis. The total prescribed dose was calculated by fraction size and the number of individual prescriptions by assigning a dose in cGy to the volumes in the DVH based on the bin size and number (i.e., bin 1 would be 0 cGy, bin 2 would be 50 cGy, bin 3 would be 100 cGy, etc.). The percent of total prescribed dose to each total region of interest (ROI) volume was then calculated. In order to verify that values calculated by the MATLAB programs were correct, calculations were replicated in Excel with the raw data. The calculated values from MATLAB agreed with the Excelgenerated values to within an average of $0.003 \%$.

\section{DVH analysis of BP dose}

The volume of BP receiving a specified dose in Grays (Vds) was computed in cc (i.e. V50 - V75). The dose in
Gray covering a specified percent of BP volume (Dvs) was also tabulated (i.e. D5 - D30). The mean, median and maximum, V50, V60, V70, V75, D5, D10, D15, D20, D25, D30 values of ipsilateral and contralateral BP were calculated based on tumor site and stage.

\section{Brachial plexus contouring technique}

The BP was contoured on both sides per the RTOGendorsed brachial plexus contouring atlas [5]. The right and left brachial plexi were contoured with a 3-5-mm diameter paint tool as separate regions of interest (ROI) in all patients. The ROIs were delineated by one radiation oncologist and reviewed and adjusted when considered appropriate by a second radiation oncologist. Figure 1 shows an example of our BP-contouring technique.

\section{Follow up}

Patients were seen in follow-up at 1 month after completion of radiation, followed by approximately every 3 months in the first year, every 4 months in the 2nd year and every 6 months for five years then with yearly followups thereafter. The first imaging study CT and or PET scan was obtained 1-3 months after treatment to assess response and thereafter as indicated. Patients usually had an imaging study performed once a year. Radiation induced brachial plexopathy was diagnosed if the affected side was within the prior radiation field and the symptoms were not due to another known etiology (i.e. tumor progression or acute injury). All adverse events were retrospectively reported based on the Common Terminology Criteria for Adverse Events version 3.0 (CTCAE v3.0). Based on CTCAE v3.0 injury to the BP was graded

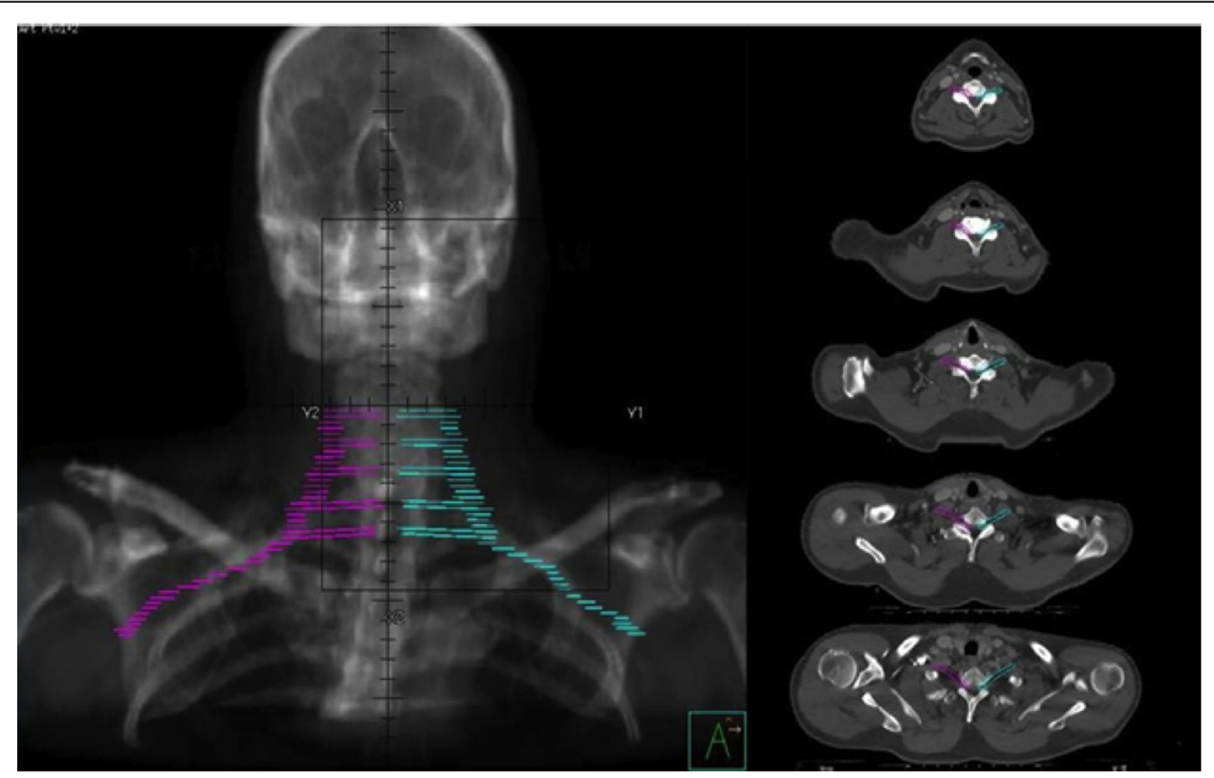

Figure 1 Digital reconstructed radiograph of contoured brachial plexus with the right BP in magenta and left BP in cyan. Right panel shows axial images of representative slices contoured with the right and left BP. 
1-5 as: grade 1 was asymptomatic, grade 2 was symptomatic not interfering with activities of daily living, grade 3 was symptomatic and interfering with activities of daily living, grade 4 was disabling and grade 5 was death. As per our institutional practice, regular follow up for HNSCC patients included evaluation of treatment-induced adverse events that were graded according to CTCAE v3.0 and reported in the patients' charts.

\section{Statistical analysis}

Dose parameters were compared across categories of clinical variables (tumor site, nodal status, stage) using analysis of variance, accounting for different levels of variation across categories. Post-hoc comparisons were done using the Tukey-Kramer method, which accounts for multiple significance testing [10]. Statistical significance was indicated when $\mathrm{p}<0.05$.

\section{Results}

\section{Patient and tumor demographics}

Table 1 lists patient and tumor characteristics for the 68 patients in the study. Notably, most patients were male (84\%) and most had stage IV disease (76.47\%). Thirty-eight percent of patients had T2 disease and 61.8\% of patients had N2 disease. The oropharynx (48.5\%) was the most commonly treated site. Fifty patients were treated definitively with the remainder treated adjuvantly and $86.8 \%$ of cases were treated with chemotherapy.

\section{Tumor site, stage and BP dose association}

The mean volume of BP was $11.6 \mathrm{cc}$ (Median, $11.6 \mathrm{cc}$ ). Table 2 shows the association between components of the DVH for both ipsilateral and contralateral BP compared across categories of clinical variables including tumor site, nodal status, and tumor group stage. For ipsilateral $\mathrm{BP}$, comparisons of DVH components across sites were significant except for volume of BP receiving > 70Gy (V70) or $>75 \mathrm{~Gy}$ (V75). When significant, these components were lowest for oral cavity and hypopharynx tumors. Comparisons of ipsilateral DVH components across nodal status were significant for all components with $\mathrm{N0}$ and N1 tumors having lower values than N2 and N3 nodes. Comparisons of ipsilateral tumors by stage of disease were significant only for V60, D10, and D20 where stage III or lower stage tumors had lower values than stage IV tumors. DVH components for the contralateral BP were significant across sites except for volume of BP receiving $>70 G y$ (V70) and $>75$ Gy (V75). When significant, oral cavity and oropharyngeal tumors had lower values than other tumors. Based on nodal status, contralateral DVH components were significant except for the maximum dose, volume of BP receiving $>70$ Gy (V70) and $>75$ Gy (V75), and when significant, showed an increase in values with increasing nodal stage. However when stage group was evaluated for
Table 1 Patient and tumor characteristics

\begin{tabular}{|c|c|c|c|}
\hline Characteristics & & & \\
\hline \multirow[t]{3}{*}{ Age (in years) } & Mean & 56 & \\
\hline & Median & 55 & \\
\hline & Range & $30-89$ & \\
\hline \multirow[t]{4}{*}{ Follow up (in months) } & Mean & 54 & \\
\hline & Median & 49 & \\
\hline & Range & $24-108$ & \\
\hline & & $\mathbf{N}$ & $\%$ \\
\hline \multirow[t]{3}{*}{ Sex } & Male & 57 & $84 \%$ \\
\hline & Female & 11 & $16 \%$ \\
\hline & Total & 68 & $100 \%$ \\
\hline \multirow[t]{5}{*}{ Group stage } & $\left.\right|^{*}$ & 1 & $1.5 \%$ \\
\hline & $\|$ & 3 & $4.4 \%$ \\
\hline & III & 8 & $11.8 \%$ \\
\hline & IV & 52 & $76.47 \%$ \\
\hline & Total & 68 & $100 \%$ \\
\hline \multirow[t]{6}{*}{ T status } & TO & 10 & $14.7 \%$ \\
\hline & $\mathrm{T} 1$ & 11 & $16.2 \%$ \\
\hline & $\mathrm{T} 2$ & 26 & $38.2 \%$ \\
\hline & T3 & 10 & $14.7 \%$ \\
\hline & T4 & 11 & $16.2 \%$ \\
\hline & Total & 68 & $100 \%$ \\
\hline \multirow[t]{5}{*}{$\mathrm{N}$ stage } & NO & 13 & $19.1 \%$ \\
\hline & N1 & 8 & $11.8 \%$ \\
\hline & N2 & 42 & $61.8 \%$ \\
\hline & N3 & 5 & $7.4 \%$ \\
\hline & Total & 68 & $100 \%$ \\
\hline \multirow[t]{7}{*}{ Primary tumor site } & Oral Cavity & 6 & $8.8 \%$ \\
\hline & Oropharynx & 33 & $48.5 \%$ \\
\hline & Nasopharynx & 9 & $13.2 \%$ \\
\hline & Hypopharynx & 3 & $4.4 \%$ \\
\hline & Larynx & 7 & $10.3 \%$ \\
\hline & Unknown Primary & 10 & $14.7 \%$ \\
\hline & Total & 68 & $100 \%$ \\
\hline \multirow[t]{3}{*}{ Aim of treatment } & Definitive & 50 & $73.5 \%$ \\
\hline & Adjuvant + Postop & 18 & $26.5 \%$ \\
\hline & Total & 68 & $100 \%$ \\
\hline \multirow[t]{3}{*}{ Chemotherapy } & Yes & 59 & $86.8 \%$ \\
\hline & No & 9 & $13.2 \%$ \\
\hline & Total & 68 & $100 \%$ \\
\hline
\end{tabular}

${ }^{*}$ Nasopharynx Squamous Cell Carcinoma.

dose parameters for the contralateral BP only mean dose, and volume of BP receiving $>50$ Gy were significantly different with lower stage tumors having lower values. 
Table 2 Association between tumor site, nodal status, group stage and BP dose

\begin{tabular}{|c|c|c|c|}
\hline $\begin{array}{l}\text { Combined BP } \\
(n=136)\end{array}$ & $\begin{array}{l}\text { SITE (OC, OPhx, NPhx, } \\
\text { Larynx, UNP, HPhx) }\end{array}$ & $\begin{array}{l}\text { N status } \\
\text { (N0 - N3) }\end{array}$ & $\begin{array}{l}\text { Group stage } \\
\text { (I-III vs. IV) }\end{array}$ \\
\hline Mean dose & 0.005 & 0.0002 & 0.037 \\
\hline Max dose & 0.0002 & 0.002 & 0.17 \\
\hline V50cc & 0.012 & $<.0001$ & 0.049 \\
\hline V60cc & 0.0003 & $<.0001$ & 0.08 \\
\hline V70cc & 0.002 & $<.0001$ & 0.33 \\
\hline V75cc & 0.19 & 0.08 & 0.039 \\
\hline D5 & 0.0005 & 0.0001 & 0.12 \\
\hline D10 & $<.0001$ & $<.0001$ & 0.026 \\
\hline D15 & 0.0006 & $<.0001$ & 0.06 \\
\hline D20 & 0.003 & $<.0001$ & 0.022 \\
\hline D25 & 0.22 & $<.0001$ & 0.10 \\
\hline D30 & 0.002 & $<.0001$ & 0.07 \\
\hline $\begin{array}{l}\text { Ipsilateral BP } \\
(\mathrm{n}=68)\end{array}$ & SITE & $\begin{array}{l}\text { N status } \\
\text { (N0 - N3) }\end{array}$ & $\begin{array}{l}\text { Group stage } \\
\text { (I-III vs. IV) }\end{array}$ \\
\hline Mean dose & $<.0001$ & $<.0001$ & 0.06 \\
\hline Max dose & 0.038 & 0.021 & 0.20 \\
\hline V50cc & 0.004 & 0.001 & 0.10 \\
\hline V60cc & 0.019 & $<.0001$ & 0.010 \\
\hline V70cc & 0.53 & $<.0001$ & 0.06 \\
\hline V75cc & 0.53 & 0.001 & 0.28 \\
\hline D5 & 0.004 & 0.0004 & 0.11 \\
\hline D10 & 0.003 & $<.0001$ & 0.007 \\
\hline D15 & 0.001 & 0.0006 & 0.08 \\
\hline D20 & 0.001 & $<.0001$ & 0.021 \\
\hline D25 & 0.013 & 0.0003 & 0.14 \\
\hline D30 & 0.0004 & 0.0004 & 0.06 \\
\hline
\end{tabular}

\begin{tabular}{llll}
\hline $\begin{array}{l}\text { Contralateral } \\
\text { BP }(\mathbf{n}=\mathbf{6 8})\end{array}$ & $\begin{array}{l}\text { SITE (OC, OPhx, NPhx, } \\
\text { Larynx, UNP, HPhx) }\end{array}$ & $\begin{array}{l}\text { N status } \\
\text { (N0 - N3) }\end{array}$ & $\begin{array}{l}\text { Group stage } \\
\text { (I-III vs. IV) }\end{array}$ \\
\hline Mean dose & $<.0001$ & 0.004 & 0.045 \\
Max dose & $<.0001$ & 0.10 & 0.29 \\
V50cc & 0.003 & 0.006 & 0.041 \\
V60cc & $<.0001$ & 0.021 & 0.44 \\
V70cc & 0.15 & 0.20 & 0.66 \\
V75cc & 0.15 & 0.86 & 0.52 \\
D5 & $<.0001$ & 0.042 & 0.21 \\
D10 & $<.0001$ & 0.030 & 0.14 \\
D15 & 0.0001 & 0.004 & 0.09 \\
D20 & $<.0001$ & 0.009 & 0.11 \\
D25 & $<.0001$ & 0.018 & 0.19 \\
D30 & $<.0001$ & 0.006 & 0.13 \\
\hline
\end{tabular}

$\mathrm{OC}=$ oral cavity, $\mathrm{OPhx}=$ oropharynx, NPhx = nasopharynx, UNP = unknown primary, $\mathrm{HPhx}=$ Hypopharynx.
Volume of brachial plexus receiving dose (cc)

For the ipsilateral BP the mean volume receiving more than 60Gy (V60) was 4.37 cc or $37.74 \%$ (median $3.92 \mathrm{cc}$ or $36 \%$ ) and $70 \mathrm{~Gy}$ (V70) was $1.47 \mathrm{cc}$ or $12.74 \%$ (median $44 \mathrm{cc}$ or $3.84 \%$ ). Whereas the mean volume to the contralateral BP receiving more than 60Gy (V60) was $2.0 \mathrm{cc}$ or $17.9 \%$ (median $0.5 \mathrm{cc}$ or $4.56 \%$ ) and $70 \mathrm{~Gy}$ (V70) was $0.43 \mathrm{cc}$ or $3.74 \%$ (median $0 \mathrm{cc}$ ). Of note the median V70Gy for the ipsilateral BP was $3.84 \%$ of the BP volume ( $0.44 \mathrm{cc}$ ), implying that at least $50 \%$ of the patients had a $<5 \%$ of the ipsilateral BP volume receiving $\geq 70 \mathrm{~Gy}$ (Table 3).

\section{Percentage of brachial plexus receiving dose (\%)}

For ipsilateral BP $(n=68)$ the mean dose delivered to $\leq 5 \%$ of the BP volume (D5) was 68.7 Gy (median $69.5 \mathrm{~Gy}$, range $45-79.25 \mathrm{~Gy}$ ), to $10 \%$ (D10) was $67.37 \mathrm{~Gy}$ (median 68.50Gy, range 35.5 - $78 \mathrm{~Gy}$ ) and to $15 \%$ (D15) was 64.98 Gy (median 61Gy, range 10.5 - 77.25 Gy). The mean and median maximum point doses (Dmax) to the ipsilateral BP were $71.31 \mathrm{~Gy}$, and 72.96 Gy respectively (range: 48.99 Gy - 78.17 Gy). The mean, median, and maximum D5\%, 10\%, 15\%, 20\%, 25\%, 30\%, as well as Dmax were also calculated for the ipsilateral and contralateral BP (Table 4). Of note the mean D5\% to the contralateral BP was 59.07Gy (median 60Gy, range $15 \mathrm{~Gy}-75 \mathrm{~Gy}$ ), and the median Dmax to the contralateral BP was 62.24 Gy (mean 61.77 Gy, range 18.79 Gy 76.16 Gy).

\section{Follow up $\&$ toxicity}

The minimum follow up was 2 years (mean 54 months; range 24-108 months) for our patient cohort. A substantial percentage of our patients received higher than recommended doses to BP due to advanced tumor stage or location without adverse events. Three patients developed $\mathrm{BP}$ symptoms. However no case was due to radiotherapy or disease progression. One patient acutely developed grade 2 weakness in the ulnar nerve distribution after being hospitalized for systemic infection. MRI in this case revealed diskitis from vertebral levels C7-T1 with evidence of a phlegmon encompassing the associated nerve roots. Another patient acutely developed grade 3 right hand weakness immediately after a traumatic motorcycle injury, with imaging revealing fracture of vertebral levels C6-C7 and significant soft tissue injury. A third patient developed grade 1 finger parasthesias with workup imaging revealing degenerative disk disease with severe cervical spinal stenosis as well as neuroformainal stenosis, corresponding to the affected dermatome.

\section{Discussion}

In spite of high dose none of the patients experienced signs or symptoms of radiation induced BP injury; we are 
Table 3 Volume of BP receiving $50 \mathrm{~Gy}, 60 \mathrm{~Gy}, 70 \mathrm{~Gy}$, and $75 \mathrm{~Gy}$

\begin{tabular}{|c|c|c|c|c|c|c|c|c|}
\hline \multirow[t]{2}{*}{ Combined BP $(n=136)$} & \multicolumn{2}{|l|}{ V50Gy } & \multicolumn{2}{|l|}{ V60Gy } & \multicolumn{2}{|c|}{ V70Gy } & \multicolumn{2}{|c|}{ V75Gy } \\
\hline & cc & $\%$ & cc & $\%$ & cc & $\%$ & cc & $\%$ \\
\hline Mean & 6.27 & 54.11 & 3.18 & 27.82 & 0.95 & 8.24 & 0.13 & 1.13 \\
\hline Median & 6.62 & 62.60 & 2.47 & 23.20 & 0.00 & 0.00 & 0.00 & 0.00 \\
\hline Maximum & 14.93 & 89.20 & 12.35 & 86.00 & 8.06 & 75.80 & 6.61 & 62.18 \\
\hline \multirow[t]{2}{*}{ Ipsilateral BP $(n=68)$} & \multicolumn{2}{|l|}{ V50Gy } & \multicolumn{2}{|l|}{ V60Gy } & \multicolumn{2}{|c|}{ V70Gy } & \multicolumn{2}{|c|}{ V75Gy } \\
\hline & cc & $\%$ & cc & $\%$ & cc & $\%$ & $c c$ & $\%$ \\
\hline Mean & 7.01 & 60.23 & 4.37 & 37.74 & 1.47 & 12.74 & 0.24 & 2.01 \\
\hline Median & 7.46 & 66.70 & 3.92 & 36.00 & 0.44 & 3.84 & 0.00 & 0.00 \\
\hline Maximum & 14.93 & 89.20 & 12.35 & 86.00 & 8.06 & 75.80 & 6.61 & 62.18 \\
\hline \multirow[t]{2}{*}{ Contralateral BP $(n=68)$} & \multicolumn{2}{|l|}{ V50Gy } & \multicolumn{2}{|c|}{ V60Gy } & \multicolumn{2}{|c|}{ V70Gy } & \multicolumn{2}{|c|}{ V75Gy } \\
\hline & cc & $\%$ & cc & $\%$ & cc & $\%$ & cc & $\%$ \\
\hline Mean & 5.53 & 48.00 & 2.00 & 17.90 & 0.43 & 3.74 & 0.03 & 0.26 \\
\hline Median & 5.49 & 49.10 & 0.50 & 4.56 & 0.00 & 0.00 & 0.00 & 0.00 \\
\hline Maximum & 12.06 & 87.80 & 11.13 & 77.60 & 7.51 & 54.70 & 1.20 & 11.71 \\
\hline
\end{tabular}

The volumes of BP in centimeters cubed (cc) and in percent volume (\%) are calculated for the mean, median and maximum doses to the combined, ipsilateral and contralateral BP.

presenting detailed DVH analysis for future study comparisons. In our study, using S-IMRT for HNSCC patients treated with or without chemotherapy, a mean dose of $68.70 \mathrm{~Gy}$ and a median dose of $69.50 \mathrm{~Gy}$ to $\leq 5 \%$ of the ipsilateral BP volume did not result in any BP injury. The mean volume of ipsilateral BP receiving a mean dose of 70Gy (V70) was 1.47 cc, 75Gy (V75) was 0.24 cc suggesting that the volume of $\mathrm{BP}$ treated to high dose is relatively small. Even though each axon in the BP functions as a serial structure, the complex, multiple anastomosing arrangements of roots, trunks, divisions, and branches provide a parallel network that could mask and potentially underrepresent symptoms from a focal lesion, while no symptomatic events were identified in this study, the possibility of asymptomatic focal injury could not be excluded.
In treatment planning, attention to the $\mathrm{BP}$ will continue to be important to minimize long-term side effects. Chen et al. [2] reported on subjective symptoms experienced by $\mathrm{H} \& \mathrm{~N}$ patients related to brachial plexopathy using a symptom questionnaire with a median follow-up of 56 months; in this study $12 \%$ of patients reported positive symptoms. Of note $40 \%$ of these patients were treated with CRT and $62 \%$ of these patients were treated with IMRT using SIB technique where the dose per fraction was as high as $2.12 \mathrm{~Gy}$. In our study, with a median follow up of 49 months, all patients were treated using S-IMRT with conventional fractionation and a maximum dose per fraction of $2 \mathrm{~Gy}$ prescribed to the target volume, with progressively shrinking field resulting in lower dose per fraction and reduced BP volume in the radiation field

Table 4 Percent of brachial plexus receiving given dose in Gray (Gy)

\begin{tabular}{|c|c|c|c|c|c|c|c|}
\hline Combined BP $(n=136)$ & Dmax (Gy) & D5\% (Gy) & D10\% (Gy) & D15\% (Gy) & D20\% (Gy) & D25\% (Gy) & D30\% (Gy) \\
\hline Mean & 66.54 & 63.85 & 62.46 & 60.17 & 59.48 & 58.23 & 56.91 \\
\hline Median & 68.73 & 65.50 & 63.50 & 61.00 & 61.80 & 59.50 & 58.00 \\
\hline Maximum & 78.74 & 78 & 77.5 & 77 & 76.50 & 76.00 & 75.5 \\
\hline Ipsilateral BP $(n=68)$ & Dmax (Gy) & D5\% (Gy) & D10\% (Gy) & D15\% (Gy) & D20\% (Gy) & D25\% (Gy) & D30\% (Gy) \\
\hline Mean & 71.31 & 68.70 & 67.37 & 64.98 & 63.53 & 62.52 & 60.90 \\
\hline Median & 72.96 & 69.50 & 68.50 & 66.25 & 63.50 & 63.50 & 62.25 \\
\hline Maximum & 78.74 & 78 & 77.5 & 77 & 76.50 & 76.00 & 75.5 \\
\hline Contralateral BP $(n=68)$ & Dmax (Gy) & D5\% (Gy) & D10\% (Gy) & D15\% (Gy) & D20\% (Gy) & D25\% (Gy) & D30\% (Gy) \\
\hline Mean & 61.77 & 59.07 & 57.78 & 55.60 & 55.03 & 53.87 & 53.04 \\
\hline Median & 62.236 & 60.00 & 59.75 & 57.50 & 57.00 & 55.00 & 54.00 \\
\hline Maximum & 76.16 & 75.00 & 74.40 & 74.00 & 73.90 & 73.50 & 73.00 \\
\hline
\end{tabular}

The dose delivered to a specified percent ( $5 \%$ or D5\%, $10 \%$ or D10\%, $15 \%$ or D15\%, $20 \%$ or D20\%, $25 \%$ or D25\%, $30 \%$ or D30\%) of BP in Gy was calculated for the mean, median and maximum doses to the combined, ipsilateral and contralateral BP. 
during treatment of PTV2 and PTV3 and observed no injury to BP in our patients. The differences in dose per fraction and spatial distribution of high dose volume between SIB and S-IMRT technique may have resulted in no injury to BP in our patients. A smaller study of 43 patients from Belgium evaluated $\mathrm{H} \& \mathrm{~N}$ cancer patients treated with CRT and found no cases of brachial plexopathy [11]. Radiation therapy in this study was delivered to $35 \%$ of patients using IMRT with the remainder of patients treated with 3-dimensional conformal radiotherapy. Of note these patients were treated with $2 \mathrm{~Gy}$ per fraction daily to $40 \mathrm{~Gy}$ followed by 1.6Gy twice a day to a total dose of 72Gy in 6 weeks. This suggests that dose per fraction may also play an important role in risk of brachial plexopathy.

The use of CRT may increase the risk of brachial plexopathy although quantifying this risk is difficult. It has been suggested that a range exists from a two-fold effect to higher [12,13]. More recently dose constraints from Amini et al. [14] showed that patients treated for superior sulcus lung cancers with definitive CRT require that a median dose to the BP be kept below 69Gy and the maximum dose to $2 \mathrm{cc}$ below $75 \mathrm{~Gy}$ to prevent brachial plexopathy. We found no significant difference in the risk of brachial plexopathy for patients treated with or without CRT although most of our patients were treated with CRT.

Over the years dose tolerance as specified from Emami et al. [15] and more recently Quantec [16] note a 5\% risk of developing radiation-induced brachial plexopathy at 5 years when one-third, two-thirds, and the whole BP, respectively is treated to 62,61 and 60 Gy; for a $50 \%$ risk at 5 years, the dose tolerances are 77, 76 and 75 Gy respectively. Recent data suggests that dose to the BP in patients undergoing IMRT for head and neck malignancies maybe higher [17]. In our study, with a minimum of 2 years follow-up and mean of 54 months, there were no events of acute or late brachial plexopathy. It may be necessary to give high doses to the BP depending on tumor location; however if lower dose per fraction is given, this may be help to prevent brachial plexopathy although longer follow up is necessary. The limitations of this study include the fact that the evidence of brachial plexopathy was determined by a retrospective review of charts. Furthermore, despite the fact that RTOG guidelines were used to contour the BP and contours were checked by a Head and Neck experienced board certified radiation oncologist, it is extremely difficult to visualize the $\mathrm{BP}$ even on a high resolution $\mathrm{CT}$ scan and this process is susceptible to over and under contouring. With the evolution of technology, the guidelines for target volume and normal structure definition continue to evolve. Velde et al. [18] recently published anatomically validated contouring guidelines for the $\mathrm{BP}$ using cadaver, $\mathrm{CT}$ and MRI imaging. The authors concluded that when the BP is outlined using their methodology $100 \%$ of the
BP was included while only $37.75 \%$ of the BP was included when RTOG guidelines were used. This will have a significant impact on dose-volume data. However, this guideline needs further validation by other investigators.

\section{Conclusion}

In this study cohort, at a minimum of two years follow up, the mean dose of $68.7 \mathrm{~Gy}$, and a median dose to $69.5 \mathrm{~Gy}$ to $\leq 5 \%$ of ipsilateral BP did not result in BP injury when patients were treated with S-IMRT technique. However, longer follow up is needed.

\section{Abbreviations}

BP: Brachial Plexus; CRT: Concomitant chemo-radiation; CT: Computed Tomography; CTCAE v3.0: Common Terminology Criteria for Adverse Events version 3.0; CTV: Clinical Tumor Volume; Dmax: Maximum point doses; DVH: Dose Volume Histograms; Dvs: Dose in Gray covering specified percent of brachial plexus volume; ECE: Extra Capsular Extension; GTV: Gross Tumor Volume; HNSCC: Head and Neck Squamous Cell Carcinoma; IMRT: Intensity Modulated Radiation Therapy; IRB: Institutional Review Board; MRI: Magnetic Resonance Imaging; OARs: Organs at risk; PTV: Planning Tumor Volume; ROI: Region of interest; RTOG: Radiation Therapy Oncology Group; SIB: Simultaneous Integrated Boost; S-IMRT: Sequential Intensity Modulated Radiation Therapy; Vds: Brachial Plexus volumes receiving a specified dose.

\section{Competing interests}

The authors declare that they have no competing interests.

\section{Authors' contributions}

BBM is the study principal investigator. TOT and TR are co-first authors who contributed equally to this work. BBM, TOT, TR, MC, IB, SS, VS and AWR planned and coordinated the study. TOT, TR and MC contoured the brachial plexus in all patients. BBM reviewed the contouring volumes and approved it. $\mathrm{IB}$ retrieved the treatment parameters. AK reviewed the initial contours. AWR run the statistical analysis. All authors read and approved the final manuscript.

\section{Authors' information}

Tarita O. Thomas and Tamer Refaat are first co-authors.

\section{Author details}

'Department of Radiation Oncology, Northwestern University, Robert H. Lurie Comprehensive Cancer Center, 251 East Huron, LC-178, Chicago, IL 60611 , USA. ${ }^{2}$ Department of Preventive Medicine, Northwestern University, Robert H. Lurie Comprehensive Cancer Center, Chicago, IL, USA. ${ }^{3}$ Department of Radiology, Northwestern University, Robert H. Lurie Comprehensive Cancer Center, Chicago, IL, USA. ${ }^{4}$ Northwestern Medicine Developmental Therapeutics Institute (NMDTI), Chicago, IL, USA. ${ }^{5}$ Department of Clinical Oncology and Nuclear Medicine, Faculty of Medicine, Alexandria University, Alexandria, Egypt.

Received: 11 February 2015 Accepted: 13 April 2015

Published online: 18 April 2015

\section{References}

1. Refaat T, Choi M, Thomas TO, Bacchus I, Agulnik M, Pelzer HJ, et al. Wholefield sequential intensity-modulated radiotherapy for local-regional advanced head-and-neck squamous cell carcinoma. Am J Clin Oncol. 2013. [Epub ahead of print]

2. Chen AM, Farwell DG, Luu Q, Vazquez EG, Lau DH, Purdy JA. Intensitymodulated radiotherapy is associated with improved global quality of life among long-term survivors of head-and-neck cancer. Int J Radiation Oncol Biol Phys. 2012;84(1):170-5.

3. Schierle C, Winograd JM. Radiation-induced brachial plexopathy: review. Complication without a cure. J Reconstr Microsurg. 2004;20(2):149-52.

4. Chen AM, Hall W, Guiou M, Mathai M, Vijayakumar S, Purdy JA. Brachial plexopathy after radiation therapy for head-and-neck cancer. Int J Radiat Oncol Biol Phys. 2009;75(3):S31-2. 
5. Hall WH, Guiou M, Lee NY, Dublin A, Narayan S, Vijayakumar S, et al. Development and validation of a standardized method for contouring the brachial plexus: Preliminary dosimetric analysis among patients treated with IMRT for head-and neck cancer. Int I Radiat Oncol Biol Phys. 2008;72(5):1362-7.

6. Truong MT, Nadgir RN, Hirsch AE, Subramaniam RM, Wang JW, Wu R, et al. Brachial plexus contouring with CT and MR imaging in radiation therapy planning for head and neck cancer. Radiographics. 2010;30:1095-103.

7. Yi SK, Hall WH, Mathai M, Dublin AB, Gupta V, Purdy JA, et al. Validating the RTOG-endorsed brachial plexus contouring atlas: An evaluation of reproducibility among patients treated by intensity-modulated radiotherapy for head and neck cancer. Int J Radiat Oncol Biol Phys. 2012;82(3):1060-4.

8. Som PM, Curtin HD, Mancuso AA. Imaging-based nodal classification for evaluation of neck metastatic adenopathy. Am J Roentgenol. 2000;174(3):837-44.

9. Chao KS, Wippold FJ, Ozyigit G, Tran BN, Dempsey JF. Determination and delineation of nodal target volumes for head-and-neck cancer based on patterns of failure in patients receiving definitive and postoperative IMRT. Int J Radiat Oncol Biol Phys. 2002;53(5):1174-84.

10. Kramer CY. Extension of multiple range tests to group means with unequal numbers of replications. Biometrics. 1956;12:309-10.

11. Platteaux N, Dirix P, Hermans R, Nuyts S. Brachial Plexopathy after chemoradiotherapy for head and neck squamous cell carcinoma. Strahlenther Onkol. 2012;9:517-20

12. Olsen NK, Recht A, Lingos TI, Abner A, Vicini F, Silver B, et al. Long-term radiation complications following conservative surgery (CS) and radiation therapy (RT) in patients with early stage breast cancer. Int J Radiat Oncol Biol Phys. 1992;23(5):915-23.

13. Olsen NK, Pfeiffer P, Johannsen L, Schrøder H, Rose C. Radiation-induced brachial plexopathy: neurological follow-up in 161 recurrence-free breast cancer patients. Int J Radiat Oncol Biol Phys. 1993;26(1):43-9.

14. Amini A, Yang J, Williamson R, McBurney ML, Erasmus Jr J, Allen PK, et al. Dose constraints to prevent radiation-induced brachial plexopathy in patients treated for lung cancer. Int J Radiat Oncol Biol Phys. 2012;82(3):e391-8.

15. Emami B, Lyman J, Brown A, Coia L, Goitein M, Munzenrider JE, et al. Tolerance of normal tissue to therapeutic radiation. Int J Radiat Oncol Biol Phys. 1991;21(1):109-22.

16. Marks $L B$, Yorke ED, Jackson A, Ten Haken RK, Constine LS, Eisbruch A, et al. Use of normal tissue complication probability models in the clinic. Int J Radiat Oncol Biol Phys. 2010;76(3):S10-9.

17. Truong MT1, Romesser PB, Qureshi MM, Kovalchuk N, Orlina L, Willins J. Radiation dose to the brachial plexus in head-and-neck intensity-modulated radiation therapy and its relationship to tumor and nodal stage. Int J Radiat Oncol Biol Phys. 2012;84(1):158-64.

18. Velde JV, Audenaert E, Bruno S, Vercauteren T, Mulliez T, Vandemaele P, et al. An anatomically validated brachial plexus contouring method for intensity modulated radiation therapy planning. Int J Radiat Oncol Biol Phys. 2013;87(4):802-8

\section{Submit your next manuscript to BioMed Central and take full advantage of:}

- Convenient online submission

- Thorough peer review

- No space constraints or color figure charges

- Immediate publication on acceptance

- Inclusion in PubMed, CAS, Scopus and Google Scholar

- Research which is freely available for redistribution 